\title{
Translocation of Pesticide Residues in Tomato, Mango and Pineapple Fruits
}

\author{
Akwasi Akomeah Agyekum ${ }^{1,2}$, George Soda Ayernor ${ }^{3}$, Firibu Kwasi Saalia ${ }^{3}$ and Betty Bediako-Amoa ${ }^{3}$ \\ 1. Radiological and Medical Sciences Research Institute, Ghana Atomic Energy Commission, Legon, Accra 00233, Ghana \\ 2. Applied Radiation Biology Centre, Ghana Atomic Energy Commission, Legon, Accra 00233, Ghana \\ 3. Department of Nutrition and Food Science, University of Ghana, Legon, Accra 00233, Ghana
}

\begin{abstract}
Pesticide residues in fruit and vegetables have become a major concern associated with chemical food safety issues. Translocation of pesticides in tomato, mango and pineapple fruits in locally produced samples was investigated using gas chromatography/mass spectrophotometer (GC/MS) procedures. Samples were fractionated with respect to distance from the skin to the core and prepared for analysis. Results showed that organochlorine, organophosphate and synthetic pyrethroid residues were translocated in all fruit samples analyzed. With respect to tomato fractions, the peels retained more residues compared to the pulp and the central core. In the chemical species, organochlorines were retained more in the peels of tomato than the other fractions of the fruit. More organophosphate and organochlorine residues were detected in pineapple peels compared to the pulp. Synthetic pyrethroid residues were evenly distributed in the pineapple fruit. In mangoes, the pulp retained more chemical residues than other fractions of the fruit. More organochlorine residues were retained in the pulp of mango than in the other fractions of the fruit. Synthetic pyrethroid residues were evenly distributed throughout the mango fruit. All the chemical species identified were translocatable across the fractional parts of all the fruits. There were significant differences in translocation and residue accumulation of pesticides among the fractional parts of all samples.
\end{abstract}

Key words: Pesticides, GC/MS, translocation, tomato, mango, pineapple.

\section{Introduction}

Pesticides comprise a wide variety of chemicals with different structures, and consequently differences in their mode of action, uptake, biotransformation and elimination do exist [1]. Translocation is the process whereby pesticides are absorbed into the plant and redistributed into the tissues. Depending on their physical or chemical properties, some pesticides may be trans-laminar (travelling short distances through the surface into the tissues) or systemic (translocation of pesticides over long distances) [2]. Exposure to pesticides through consumption of fruits is almost continuous, either as a result of direct treatment or due to environmental or cross contamination. Fruits are usually subjected to pre and post-harvest treatments.

Corresponding author: Akwasi Akomeah Agyekum, M.Sc., research fields: residues and contaminants in food, chemical migrants in food. E-mail: agyekumakwasiakomeah@gmail.com.
Organophosphates, carbamates and pyrethroids are routinely applied to fruit crops for broad spectrum insect control [3, 4]; organochlorines and other compounds are mainly used as post-harvest treatments for fungi control, especially in fruits intended for direct human consumption [5].

Pesticides residues may be translocated through other mechanisms: via the apoplast or symplast or across the mesophyll into the phloem [6]. The kind of chemical residues left on the crop depends on the nature of the pesticide, the pre-harvest interval and the conditions under which the crops are stored. Translocation of pesticides can be upward or lateral (acropetal) or downward (basipetal), but upward movement is more common, particularly because many pesticides are applied to the soil [6]. Pesticides can be translocated or diffused into plant tissues depending on their chemical properties of the chemical, the time 
elapsed since the chemical was applied, conditions of crop storage as well as the morphology of the plant. Subsequently, interest on pesticide toxicity has particularly increased over the past years owing to increasing evidence of carcinogenic, mutagenic and teratogenic effects in experimental animals and exposed humans [7]. Furthermore, the usage of these chemicals has occasionally been accompanied by risk to human health and the environment because of their toxic potential, high persistence and bioaccumulation [8]. The objective of this study was to investigate the translocation of chemical pesticides across the fractional parts of locally produced tomato, pineapple and mango fruits.

\section{Materials and Methods}

\subsection{Sampling and Preparation}

Freshly harvested pineapples were sampled from three commercial farms located in the Bawjiase district of Ghana. Mangoes were sampled from three commercial farms in the Yilo district. Tomatoes were sampled from three commercial farms located in the Ada district. After stones and stalks were removed, samples were fractionated into peel, core and edible portion and analyzed accordingly. Tomatoes, pineapple and mango were given three treatments: edible portion, peel and core. Extreme care was taken to prevent cross contamination of residues from one part of the fruit to the other.

\subsection{Extraction of Samples}

Sample preparation, extraction, clean-up and analysis were carried out according to the procedure described in the Netherlands analytical methods for pesticides residues and foodstuffs with slight modifications [9].

Samples were thoroughly shredded and blended to maintain homogeneity based on treatments. Sub-sample of about $20.0 \mathrm{~g}$ was weighed and macerated with $40 \mathrm{~mL}$ ethyl acetate for $30 \mathrm{~s}$. To adjust acidity and remove moisture, $5.0 \mathrm{~g}$ sodium hydrogen carbonate and $20.0 \mathrm{~g}$ anhydrous sodium sulfate, respectively, were then added, and further macerated for $90 \mathrm{~s}$ using the ultra-turrax macerator. The samples were then centrifuged for $5 \mathrm{~min}$ at $3,000 \mathrm{rpm}$ to separate the phases. The extraction process was followed by a clean-up step using solid phase extraction with florisil pre-packed cartridge. The florisil $(1,000 \mathrm{mg} / 6 \mathrm{~mL})$ cartridge was conditioned with $10 \mathrm{~mL}$ ethyl acetate. Aliquot of the organic layer extract were loaded onto the cartridge, and eluted with $10 \mathrm{~mL}$ ethyl acetate afterwards.

\subsection{Determination/Quantification of Synthetic} Pyrethroid, Organochlorine and Organophosphate Pesticides

Quantification of synthetic pyrethroid, organochlorine and organophosphate pesticides were performed using a Varian CP-3800 GC/MS with a Varian CP-8400 autosampler with an analytical gas column $(30 \mathrm{~m}+10 \mathrm{~m})$ EZ guard $0.25 \mathrm{~mm}$ internal diameter fused silica capillary coated with VF- $5 \mathrm{~ms}$ (0.25 $\mu \mathrm{m}$ film thickness). Aliquots of $1-2 \mu \mathrm{L}$ were injected into the GC/MS. The oven temperature was programmed at $80-180{ }^{\circ} \mathrm{C}$ at a rate of $25{ }^{\circ} \mathrm{C} / \mathrm{min}$ for synthetic pyrethroids, $70-200{ }^{\circ} \mathrm{C}$ for organophosphates and $70{ }^{\circ} \mathrm{C}$ for $2 \mathrm{~min}$, ramp at $25^{\circ} \mathrm{C} / \mathrm{min}$ to $180{ }^{\circ} \mathrm{C}$ and finally ramp at $5{ }^{\circ} \mathrm{C} / \mathrm{min}$ to $300^{\circ} \mathrm{C}$, held for $2 \mathrm{~min}$

Injector temperature was $250{ }^{\circ} \mathrm{C}$ and the helium flow rate was $1.3 \mathrm{~mL} / \mathrm{min}$ constant flow. The residue levels of organochlorines/organophosphate/pyrethroid pesticides were quantitatively determined by the external standard method using peak area. Measurement was carried out within the linear range of the detector. The peak areas whose retention times coincided with the reference standards were extrapolated on their corresponding calibration curves to obtain their concentration.

\subsection{Quality Control and Quality Assurance}

All reagents used during the analysis were exposed to the same extraction procedures. In all batches of 
organophosphate/organochlorine/synthetic pyrethroids pesticides residues analysis, reagent blanks, procedural matrix blanks and triplicate samples were included. For the reagent blanks in each extraction procedure, no organochlorine/synthetic pyrethroids pesticides were detected. All extracts were kept frozen until quantification was achieved.

Recalibration curves were run with each batch of samples to check that the correlation coefficient was kept at $R^{2}=0.99$ or better. The method used was an international method, optimized and validated using various agricultural products [10]. A fortification level of $0.05 \mathrm{mg} / \mathrm{kg}$ of standard mixtures was chosen before analysis to evaluate the recovery of compounds in the fresh produce samples analyzed. Fortified samples were determined with good recoveries. The recovery of organochlorine/synthetic pyrethroids pesticides residues ranged between $80 \%$ and $120 \%$ for most of the organochlorine/synthetic pyrethroids pesticides, and it was calculated as Eq. (1):

Recovery $(\%)=[($ concentration of pesticide recovered from fortified sample) $\times$ 100] /(concentration of pesticide added to sample)

\subsection{Limit of Detection}

Detection limits of the method were also assessed based on the lowest concentrations of the residues in each of the matrices that could be reproducibly measured at the operating conditions of the GC, which were $0.001 \mathrm{mg} / \mathrm{kg}$. Blank analyses were also carried out in order to check any interfering species in the reagents [11].

\subsection{Reagents}

Individual certified reference standards of organophosphates, organochlorines and synthetic pyrethroids used for the identification and quantification were obtained from Dr. Ehrenstorfer GmbH (Augsburg, Germany). Pesticide grade ethyl acetate and analytical grade acetone were obtained from British Drug House Chemicals (BDH Chemicals), England. Anhydrous sodium hydrogen carbonate and sodium sulfate were analytical grade obtained from Aldrich (Chemie, Germany). Solid phase extraction Envi-carb/LC-NH $2(500 \mathrm{mg} / 6 \mathrm{~mL})$ was from Supelco and florisil $(1,000 \mathrm{mg} / 6 \mathrm{~mL})$ was from Phenomenex.

\section{Results and Discussion}

\subsection{Translocation of Pesticides in Tomatoes}

Fig. 1 depicts the distribution of pesticide residues across the fractional parts of tomatoes. The chemicals: fonofos, beta-hexachlorocyclohexane $(\beta-\mathrm{HCH})$ and endosulfan sulfate were predominantly found in the tomato sample. Dieldrin, fenitrothion, chlorpyrifos,

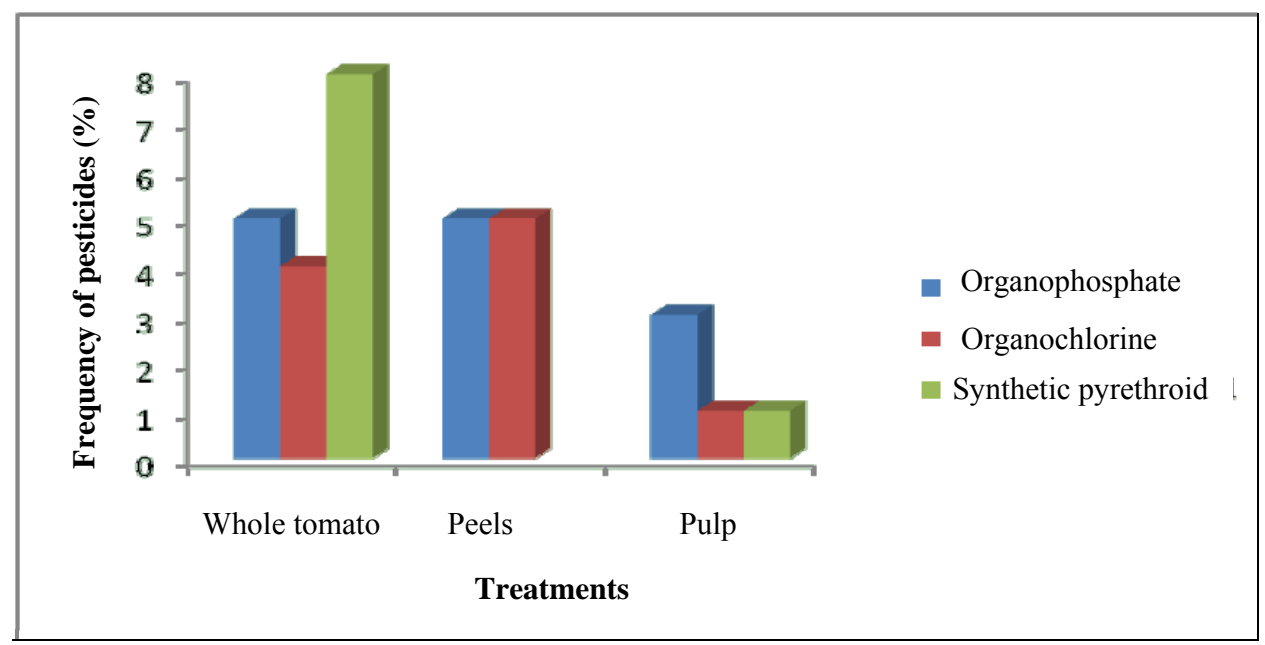

Fig. 1 Distribution of pesticide residues across fractional parts of tomatoes. 
lindane and p,p'-dichlorodiphenyltrichloroethane (p,p'-DDT) were found mainly in the tomato peels.

The peels retained more of organophosphate and organochlorine residues than the other fractions of the fruit. Whole tomato had traces of the synthetic pyrethroids (cypermethrin, lambda cyhalothrin, allethrin, cyfluthrin I, cyfluthrin II, cyfluthrin III, cyfluthrin IV and bifenthrin). Chlorpyrifos and fenitrothion were found in the peels of tomato. The peels were found to have the highest level of organochlorine pesticide residues.

The absence of fonofos and fenitrothion in whole tomato and subcuticular samples may be attributed to the dilution of the chemical. The chemical may also be in trace forms undetected by the instrument. Even though the farmers claimed dichlorodiphenyltrichloroethane

p,p'-dichlorodiphenyldichloroethylene (p,p'-DDE) and p,p'-dichlorodiphenyldichloroethane (p,p'-DDD) was not applied, the metabolites of DDT were found in the crop samples. This indicates that DDT might have been used in previous agricultural seasons as corroborated by Mbakaya et al. [12]. Essentially, pesticide residues in food and crops are a result of application of pesticides to agricultural field and contaminated soils. Moreover, sometimes few farmers do not wait long enough for the residues to wash off after spraying before harvesting because of their high demand for farm products and low perception of the toxic effects of pesticide residues on food [13].

High organochlorine levels in the peels of tomato may be attributed to the high carotenoid levels which are responsible for retaining chlorinated hydrocarbons in the peels of fruit and vegetables [14]. Pesticide residues can be translocated internally, e.g., cucumber and garlic sprouts; thus, washing would not reduce pesticide residues very easily [15].

Whole tomato samples had the highest level of synthetic pyrethroid contamination. The occurrence of fonofos, $\beta-\mathrm{HCH}$, parathion and endosulfan sulfate in the whole tomato samples suggest the hydrophilic nature of these pesticides. The hydrophobic pesticides were predominantly found in the peels and the hydrophilic pesticides were found in the whole tomato samples. Most of the organochlorine pesticides identified in the study were persistent in agricultural systems which is consistent with work done by Mbakaya et al. [12], who reported that organochlorines are resistant to environmental degradation, which allows them to accumulate in plant and animal tissues to become concentrated in the upper part of the food pyramid. The persistence of pesticides in plants are influenced by characteristics, such as the plant species involved, the nature of the harvested crop, the structure of the cuticle, the stage and rate of growth and the general condition of the plant [2]. The absence of synthetic pyrethroid in peels may be due to the fact that pyrethroids are usually broken down by sunlight and the atmosphere in one or two days, and has negligible persistence. Rate of pesticide penetration, metabolism and phytotoxicity may differ among plant species and thus may influence distribution patterns [16].

\subsection{Pesticide Translocation in Pineapple}

Fig. 2 shows the distribution of pesticides residues across the fractional parts of pineapple. The chemicals: lindane, delta-hexachlorocyclohexane $(\delta-\mathrm{HCH})$, fonofos and dieldrin were predominantly found in the peel fractions. Aldrin was mainly found in the pulp samples. Methamidophos and parathion were found predominantly in the peel and whole pineapple samples.

The peel and whole pineapple fractions retained more of the organophosphate chemical residue. Fonofos was predominantly found in the peel fractions and can be ascribed to the non-polar nature of the pesticide. The absence of fonofos in whole pineapple and pulp samples may be attributed to dilution of the pesticide. The chemical may also be in trace forms undetected by the instrument. More of the organochlorine pesticides were detected in the peels. 


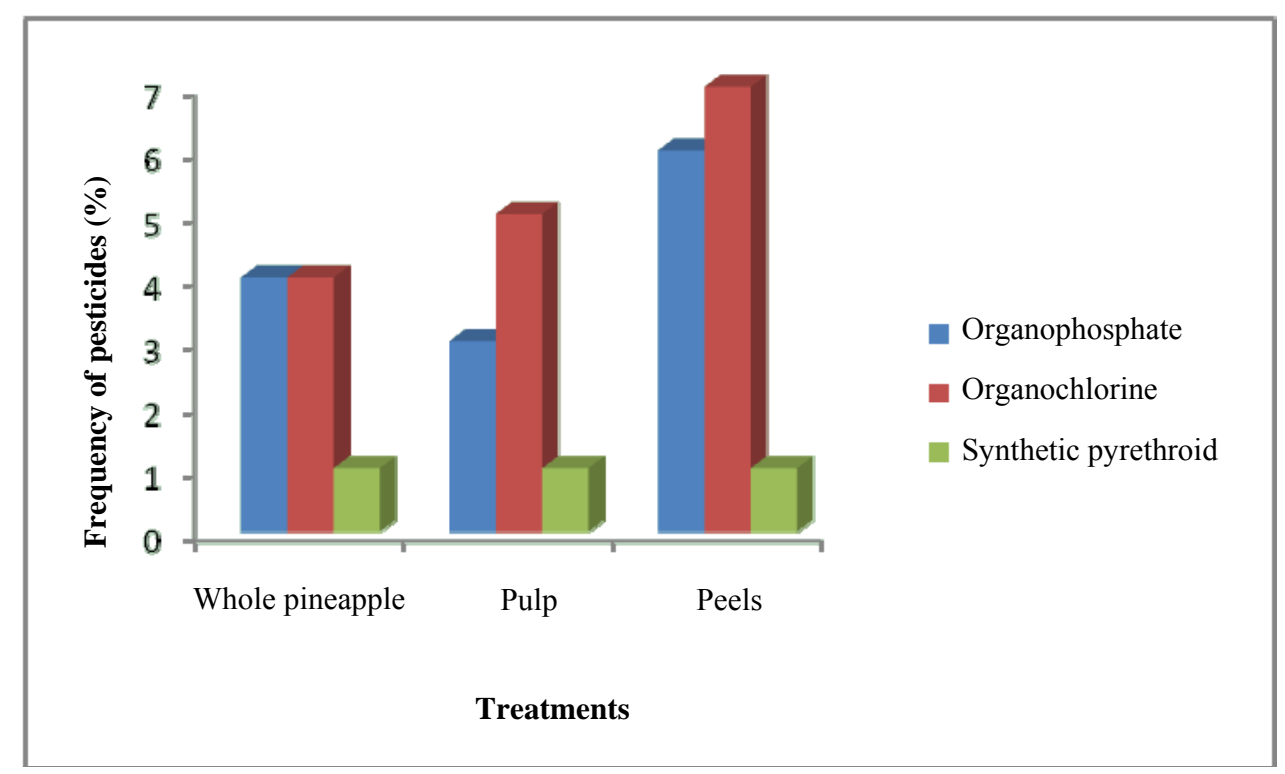

Fig. 2 Distribution of pesticide residues across fractional parts of pineapple.

The chemicals: aldrin, $\delta$ - $\mathrm{HCH}, \beta-\mathrm{HCH}, \gamma$-chlordane, heptachlor, $\beta$-endosulfan, endosulfan sulfate and lindane are persistent in soils, global transport, distribution and toxicity and their occurrence could be due to previous use in agricultural systems [10]. This may be due to the hydrophobic nature of the organochlorine pesticides. The presence of detectable residues of hexachlorobenzene (HCB) in samples may indicate that $\mathrm{HCB}$ has been released into the environment as a by-product from industrial processes and waste incineration [17] rather than from its agricultural use as a fungicide. $\beta-\mathrm{HCH}$ and $\delta-\mathrm{HCH}$ isomers detected in most of the samples indicates that technical $\mathrm{HCH}$ and lindane have been used widely. It may also be attributed to the persistent nature of these chemicals which are lipophilic in nature and may move long distances in surface runoff or groundwater thereby contaminating water bodies which are used for irrigating horticultural crops [14]. Although dieldrin did not appear to be used by farmers in the horticultural sector, their occurrence in samples is not surprising. This is because dieldrin enters the environment through manufacturing emissions [18, 19]. The equal level of the bifenthrin across the fractional parts of pineapple suggests that bifenthrin is not translocated to any significant extent when applied to plants. The trace of chemical which is translocated, is a metabolite of the acidic half of the molecule.

The absence of fonofos and fenitrothion in whole tomato and subcuticular samples may be attributed to the dilution of the chemical. The chemical may also be in trace forms undetected by the instrument. Even though the farmers claim DDT was not applied, the metabolites of DDT were found in the crop samples. This indicates that DDT might have been used in previous agricultural seasons as upheld by Mbakaya et al. [12]. It also suggests the biotransformation of the parent materials in the plant systems to DDE and DDD or old sources of DDT contamination.

High organochlorine levels in the peels of tomato may be attributed to the high carotenoid levels which are responsible for retaining chlorinated hydrocarbons in the peels of fruit and vegetables. This is in accord with work done by Miglioranza et al. [14].

\subsection{Translocation of Pesticide in Mango}

Fig. 3 shows the distribution of pesticide residues in fractional parts of mango. Peel and whole mango fractions retained more of the organophosphate pesticide residue. More of the organochlorine pesticide residues were found in the pulp fraction. The 
peel and whole mango samples had equal numbers of chemical pesticides identified. This suggests that these pesticides have the ability to penetrate through the surface into the plant tissues. Thus moderately to highly lipophilic pesticide residues are confined to the outer surfaces. The presence of lindane, $\delta-\mathrm{HCH}$, p,p'-DDE, p,p'-DDD, endrin, kresoxim methyl and $\gamma$-chlordane in pulp samples could suggest the mobility of these pesticides into the deep layers of the plant tissue. The presence of p,p'-DDE and p,p'-DDD in samples analyzed indicate that DDT might have been used in previous agricultural production. The presence of detectable levels of $\delta$ - $\mathrm{HCH}$ and $\beta-\mathrm{HCH}$ indicate that technical $\mathrm{HCH}$ has been widely used or may have been released into the environment as a by-product from industrial processes and waste incineration rather than from its agricultural use as a fungicide [17]. Lindane is much less bioaccumulative than the other organochlorine pesticides because of the fairly low lipophilicity and short half-life in biota. Moreover, the chemical properties of lindane also show that this chemical is easily evaporated, and transported through air and water to long distances [20]. Vegetables on the Ghanaian market were found to contain detectable levels of chlopyrifos, lindane, endosulfan, lambda-cyhalothrin, and DDT residues in lettuce, cabbage, tomato and onion [21-23]. It may also be attributed to the persistent nature of these chemicals which are lipophilic in nature and may move long distances in surface runoff or groundwater, thereby contaminating water bodies that are used for irrigating most horticultural crops. This data therefore corroborates the findings of Osman et al. [24] who suggested that some vegetable samples contained more residues, and this might be that vegetables cultivated are highly sensitive to pests and hence require successive applications of pesticides.

The equal level of synthetic pyrethroid residues in mango across the fractional parts of the produce suggest that pyrethroids are not translocated to any significant extent when applied to plants. The chemicals: endosulfan sulfate, lindane, $\gamma$-chlordane, $\delta$-HCH, p,p'-DDD and p,p'-DDE were predominantly found in the pulp fraction. Fonofos, methoxychlor and $\beta-\mathrm{HCH}$ were most retained in the peels fractions. Farmers are mostly unaware of safe use of pesticides thereby have a tendency to apply over-dose of pesticides to produce a high quality fruit and vegetables.

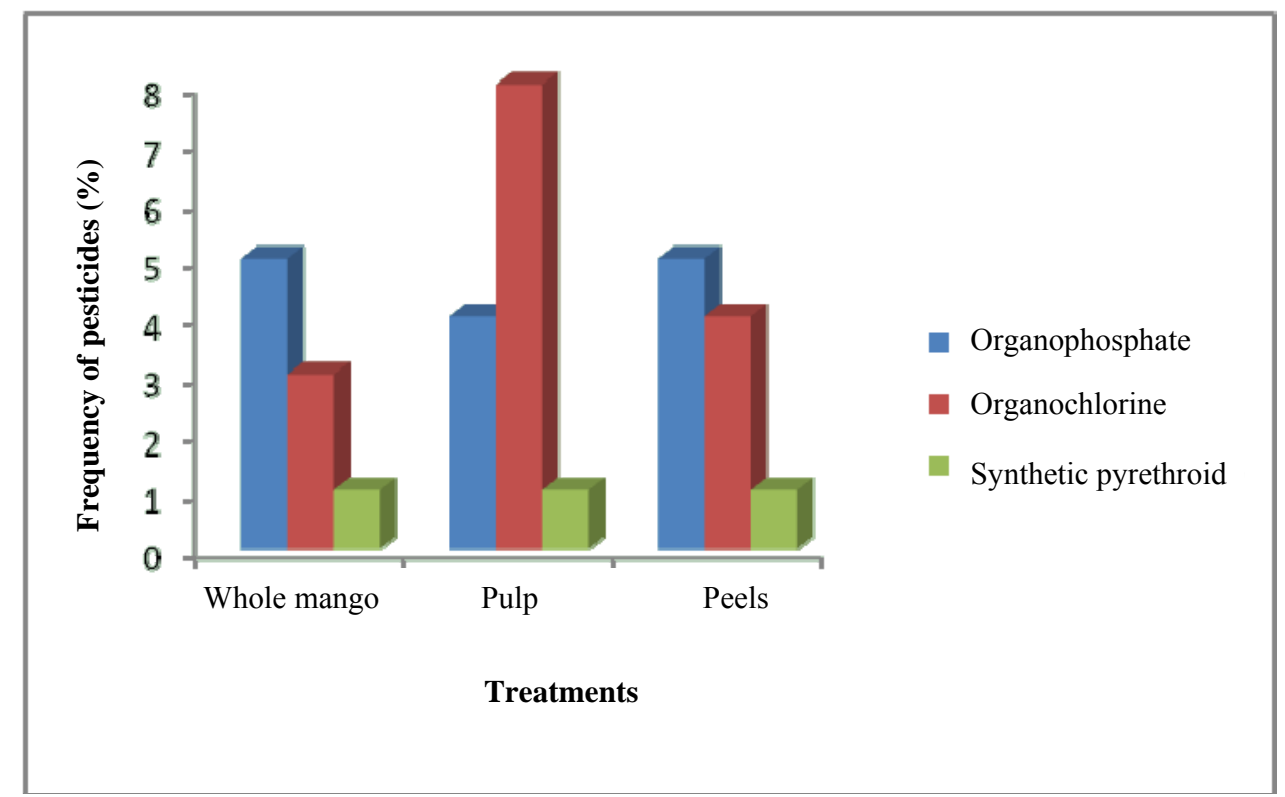

Fig. 3 Distribution of pesticide residues across the fractional parts of mango. 


\section{Conclusions}

In the present study, translocation of pesticide residues in fruits was evaluated. Through translocation, pesticide residues were found across the fractional parts of tomatoes, mango and pineapple, and dieldrin, fenitrothion, chlorpyrifos, lindane and p,p'-DDT were found mainly in the tomato peels. Methamidophos and parathion were found predominantly in the peel and edible portions of the pineapple samples. It is recommended that a combination of washing and cooking should effectively remove most of the residues on/in food samples. In view of the number of positive detections, it is imperative to conduct an extensive study monitoring fruits grown in different geographical regions of Ghana taking into consideration seasonal variations.

\section{Acknowledgments}

The authors gratefully thank the collaboration of the staffs of the Pesticide Residue Laboratory and the Head of the Food and Agriculture Laboratories of the Ghana Standards Authority.

\section{References}

[1] Younes, M., and Galal-Gorchev, H. 2000. "Pesticides in Drinking Water-A Case Study." Food Chem. Toxicol. 38 (1): 87-90.

[2] Edwards, C. A. 1975. "Factors that Affect the Persistence of Pesticides in Plants and Soils." Pure Appl. Chem. 42 (1-2): 39-56.

[3] Rawn, D. F., Roscoe, V., Krakalovich, T., and Hanson, C. 2004. "N-Methyl Carbamate Concentrations and Dietary Intake Estimates for Apple and Grape Juices Available on the Retail Market in Canada." Food Additives and Contaminants 21 (6): 555-63.

[4] Rawn, D. F., Quade, S. C., Shields, J. B., Conca, G., Sun, W. F., Lacroix, G. M., Smith, M., Fouquet, A., and Bélanger, A. 2006. "Organophosphate Levels in Apple Composites and Individual Apples from a Treated Canadian Orchard." Journal of Agricultural and Food Chemistry 54 (5): 1943-8.

[5] Park, D. S., Peterson, C., Zhao, S., and Coats, J. R. 2004. "Fumigation Toxicity of Volatile Natural and Synthetic Cyanohydrins to Stored-Product Pests and Activity as Soil Fumigants." Pest Management Science 60 (8): 833-8.
[6] Ebeling, W. 1963. "Analysis of the Basic Processes Involved in the Deposition, Degradation, Persistence and Effectiveness of Pesticides." Residue Reviews 3: 128-35.

[7] Tahir, M. U., Naik, S. I., Rehman, S., and Shahzad, M. 2009. "A Quantitative Analysis for the Toxic Pesticide Residues in Marketed Fruits and Vegetables in Lahore, Pakistan." Biomedica 25: 171-4.

[8] Bempah, C. K., Donkor, A., Yeboah, P. O., Dubey, B., and Osei-Fosu, P. 2011. "A Preliminary Assessment of Consumer's Exposure to Organochlorine Pesticides in Fruits and Vegetables and the Potential Health Risk in Accra Metropolis, Ghana." Food Chemistry 128 (4): 1058-65.

[9] Ministry of Public Health, Water and Sport. 1996. Netherlands Analytical Methods for Pesticide Residues in Foodstuffs, 6th ed.. The Netherlands: General Inspectorate for Health Inspection, Ministry of Public Health, Water and Sport.

[10] Chung, S. W. C., and Chan, B. T. P. 2010. "Validation and Use of a Fast Sample Preparation Method and Liquid Chromatography-Tandem Mass Spectrometry in Analysis of Ultra-Trace Levels of 98 Organophosphorus Pesticide and Carbamate Residues in a Total Diet Study Involving Diversified Food Types.” J. Chromatogr. A 1217 (29): 4815-24.

[11] Gilbert-López, B., García-Reyesa, J. F., Fernández-Alba, A. R., and Molina-Díaz, A. 2010. "Evaluation of Two Sample Treatment Methodologies for Large-Scale Pesticide Residue Analysis in Olive Oil by Fast Liquid Chromatography-Electrospray Mass Spectrometry." J. Chromatogr. A 1217 (24): 3736-47.

[12] Mbakaya, C. F., Ohayo-Mitoko, G. J., Ngowi, V. A., Mbabazi, R., Simwa, J. M., Maeda, D. N., Stephens, J., and Hakuza, H. 1994. "The Status of Pesticide Usage in East Africa." African Journal of Health Sciences 1 (1): $37-41$.

[13] Amoah, P., Drechsel, P., Abaidoo, R. C., and Ntow, W. J. 2006. "Pesticide and Pathogen Contamination of Vegetables in Ghana's Urban Markets." Arch. Environ. Conta. Toxicol. 50 (1): 1-6.

[14] Miglioranza, K. S. B., Aizpin-Moreno, J. E., Moreno, V. J., Osterrieth, M. L., and Escalante, A. H. 1999. "Fate of Organochlorine Pesticides in Soils and Terrestrial Biota of 'Los Padres' Pond Watershed, Argentina." Environ. Pollut. 105 (1): 91-9.

[15] Krol, W. J., Arsenault, T. L., Pylypiw, H. M., and Mattina, M. J. I. 2000. "Reduction of Pesticide Residues on Produce by Rinsing." Journal of Agricultural and Food Chemistry 48 (10): 4666-70.

[16] Bromilow, R. H., Chamberlain, K., and Evans, A. A. 1990. "Physicochemical Aspects of Phloem Translocation of Herbicides.” Weed Sci. 38 (3): 305-14. 
[17] Van Birgelen, A. P. 1998. "Hexachlorobenzene as a Possible Major Contributor to the Dioxin Activity of Human Milk." Environ. Health Perspect. 106 (11): 683-8.

[18] Crosby, D. G., and Moilanen, K. W. 1974. "Vapour-Phase Photodecomposition of Aldrin and Dieldrin." Arch. Environment. Contam. Toxicol. 2 (1): 62-74.

[19] Majewski, M. S., and Capel, P. D. 1995. Pesticides in the Atmosphere: Distribution, Trends and Governing Factors (Pesticides in the Hydrologic System). Chelsea, Mich., USA: Ann Arbor Press.

[20] Sun, F., Wong, S. S., Li, G. C., and Chen, S. N. 2006. "A Preliminary Assessment of Consumer's Exposure to Pesticide Residues in Fisheries Products." Chemosphere 62 (4): 674-80.
[21] Ntow, W. J. 2001. "Organochlorine Pesticides in Water, Sediment, Crops and Human Fluids in a Farming Community in Ghana." Environmental Contamination and Toxicology 40 (4): 557-63.

[22] Ntow, W. J. 1998. "Pesticide Misuse at Akumadan to Be Tackled." NARP Newsletter 3 (3): 25.

[23] Armah, A. K., Dapaah, G. A., and Wiafi, G. 1999. "Water Quality Studies on Two Irrigation Associated Rivers in Southern Ghana." Journal of Ghana Science Association 1 (2): 100-9.

[24] Osman, K. A., Al-Humaid, A. M., Al-Rehiayani, S. M., and Al-Redhaiman, K. N. 2010. "Monitoring of Pesticide Residues in Vegetables Marketed in Al-Qassim Region, Saudi Arabia." Ecotoxicology and Environmental Safety 73 (6): 1433-9. 